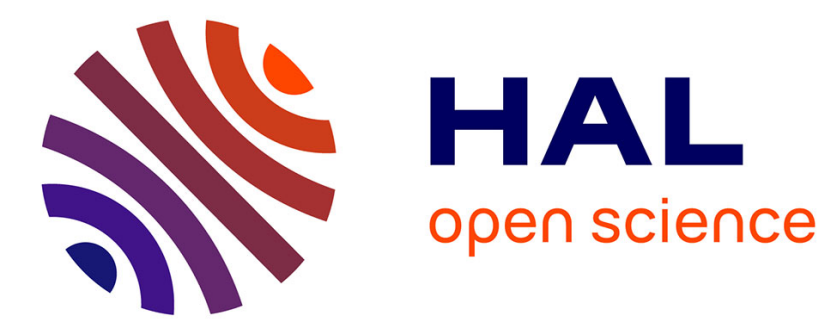

\title{
THEORETICAL ASPECTS OF NEUTRON MAGNETIC FORM FACTORS
}

\author{
B. Harmon
}

\section{To cite this version:}

B. Harmon. THEORETICAL ASPECTS OF NEUTRON MAGNETIC FORM FACTORS. Journal de Physique Colloques, 1982, 43 (C7), pp.C7-177-C7-185. 10.1051/jphyscol:1982726 . jpa-00222333

\section{HAL Id: jpa-00222333 https://hal.science/jpa-00222333}

Submitted on 1 Jan 1982

HAL is a multi-disciplinary open access archive for the deposit and dissemination of scientific research documents, whether they are published or not. The documents may come from teaching and research institutions in France or abroad, or from public or private research centers.
L'archive ouverte pluridisciplinaire HAL, est destinée au dépôt et à la diffusion de documents scientifiques de niveau recherche, publiés ou non, émanant des établissements d'enseignement et de recherche français ou étrangers, des laboratoires publics ou privés. 
JOURNAL DE PHYSIQUE

Colzoque C?, supplément au $n^{\circ} 12$, Tome 43, décembre 1982

page $\mathrm{C} 7-177$

\title{
THEORETICAL ASPECTS OF NEUTRON MAGNETIC FORM FACTORS
}

\author{
B.N. Harmon \\ Ames Laboratory-USDOE and Department of Physics, Iowa State University, \\ Ames, Iowa 50011, U.S.A.
}

\begin{abstract}
Résumé.- On prësente une revue des calculs de densités magnétiques dans les solides. Si le modèle atomique reste utile comme première approximation, il est nécessaire de faire appel aux mēthodes plus élaborées de calculs de structure de bandes pour obtenir d'une maniêre $a b$ initio les densités de spin dans les solides. On discute les calculs des contributions orbitales induites par un champ externe ou dues au couplage spin-orbite.
\end{abstract}

\begin{abstract}
The calculation of magnetization densities in solids is reviewed. The atomic model is still a useful first approximation while the more involved band structure approach is required for first principle calculations of solid state spin densities. The calculation of orbital contributions, either induced by an applied field or caused by spin-orbit coupling, is discussed.
\end{abstract}

1. Introduction.- Magnetism in solids is one of the oldest and still active topics in physics. It is a form of symmetry breaking with an energy scale typically much less than the electronic Coulomb interactions which determine the underlying material structure. Thus the magnetic properties of materials are often subtle and present a continuing challenge to both theory and experiment. Indeed the calculation of magnetization densities has been largely stimulated by experimental measurements using polarised neutrons. The experiments yield the neutron magnetic scattering amplitude arising from the interaction of the neutron's magnetic moment with the orbital currents and unpaired electron spins of the crystal. The scattering amplitude is proportional to the Fourier transform of the magnetization density. Early magnetic form factor experiments were made on elements with fairly Iocalized magnetic moments and could be analyzed using magnetization densities calculated for free ions or atoms. The atomic model is most suitable for solids containing rare earth ions with their highly localized and well shielded $4 \mathrm{f}$ orbitals. The theory based on the atomic model is we11 developed and is discussed in Section II. For crystals in which the valence electrons are responsible for the magnetization there are significant solid state effects which have been detected by highly precise polarized neutron scattering experiments. In this respect neutrons have an advantage over $X$-rays since the scattering is dominated by just those states which are interacting with the crystal environment. In Section III we discuss the case of magnetically ordered $d$-band metals where the theoretical analysis is frequently made in terms of a spin density obtained from band structure calculations and a small orbital contribution is estimated from the atomic model. For metals in which the magnetization is induced by an applied field the orbital contribution is frequently dominant, and it is in this area that there remains many questions in spite of the impressive efforts which have gone into developing expressions for the total magnetic susceptibility. In Section IV we review the present situation for the calculation of the static magnetic response in metals and express the hope that this field will see continued advances. We shall not in this review discuss the dynamical response, however this is an exciting topic which will 
surely receive much more attention once the polarized detectors at the new pulsed sources are in full operation.

II. The atomic mode1.- For most magnetic systems of interest the magnetization involves easily identifiable valence orbitals and a comparison with the corresponding atomic form factor gives valuable information about the relative expansion or contraction of these orbitals in the solid. Thus the theoretical atomic form factors, which have been tabulated, aid in the first step of data analysis by providing a scale to estimate solid state effects. As an example, the experimental magnetic form factor for ferromagnetic transition metals can be fit using the functional form [1]:

$$
f(\vec{G})=(2 / g)(1+\alpha) f_{\text {spin }}^{a}(\vec{G})+\frac{(g-2)}{g} f_{o r b i t}^{a}(\vec{G})-\alpha \delta(\vec{G})
$$

where $f_{s p i n}^{a}$ and $f_{\text {orbit }}^{a}$ are obtained from spin-polarized Hartree-Fock calculations for the free atoms [2] evaluated at the scattering vector $\vec{G}$. The $g$ factor, defined as the ratio of the magnetic moment to the spin, can be determined [3] from the gyromagnetic ratio measured in Einstein-de Haas experiments [4] and allows the separation of orbital and spin contributions. The factor $\alpha$ which was introduced to improve the fit has been ascribed to a uniform negative spin density arising from free-electron like states $[1,5]$. However, there has been some new analysis which suggests the data is consistent with $\alpha=0$ $[6,7]$. Core polarizaton and asymetric ( $e_{g}$ vs $t_{2 g}$ ) contributions can be explicitly included resulting in a nearly perfect fit for the $3 d$ transition metals. It is doubtful that further development of the atomic model for these metals would be worthwhile since solid state effects are larger than the remaining errors in the fit. The real theoretical challenge is the determination of the form factor entirely from first principles using a solid state rather than an atomic approach. Before dealing with this subject the application of the atomic model to rare earth ions should be mentioned since the model is most appropriate for the $4 \mathrm{f}$ states in these systems and has reached a mature and elegant stage.

Shortly after the discovery of the neutron Bloch [8] and Schwinger [9] showed that magnetie scattering of neutrons should be an observable effect. A few years later Halpern and Johnson [10] published a classic paper dealing with the magnetic scattering from the unpaired spin density in atoms. Some fourteen years later Trammel [11] extended their work to atoms with orbital magnetic moments using the formalism of Condon and Shortly. In 1966 Johnston applied the tensor techniques of Racah $[12,13]$ to the problem. His work was extended in a number of articles [13-18] and has been reviewed in the book by Marshall and Lovesey [19]. More recently Stassis and Deckman [20-21] have provided an elegant and more general treatment using tensor techniques by exploiting the analogy with the interaction between polarized electromagnetic radiation and atoms. They give the following expression for the magnetic scattering amplitude:

$f(\vec{q})=\gamma r_{o} \vec{\sigma} \cdot\left[\sum_{k m}\left(\frac{8 \pi}{2 k+1}\right) \frac{1}{2}\left\{\vec{x}_{k m}(q)\left\langle k\left|T_{k m}^{(e)}\right| i\right\rangle-i\left(\vec{q}_{k} \times \vec{X}_{k m}^{*}(\vec{q})\right)\left\langle f\left|T_{k m}^{(m)}\right| i\right\rangle\right\}\right]$

where $\gamma=-1.91$ is the neutron magentic moment in nuclear magnetons, $r_{o}$ is the classical electron radius, $\sigma$ is the neutron Pauli-spin matrix, the $\overrightarrow{\mathrm{x}}_{\mathrm{km}}$ are vector spherical harmonics, and $\mathrm{T}_{\mathrm{km}}^{(\mathrm{e})}, \mathrm{T}_{\mathrm{km}}^{(\mathrm{m})}$ are the irreducible tensor operators corresponding to the electronic and magnetic multipoles of the system. For atoms the multipole operators are expressed as products of radial integrals and matrix elements of Racah tensors which are related to the generators used in the classification of atomic states. Symmetry properties can therefore be easily identified and exploited. The formalism is gauge invarient by including the electric multipole moments although they are not important for elastic scattering. For future inelastic experiments at pulsed source energies or for mixed valent materials where parity changes between initial and final states may occur for sma11 energy transfer the electric multipole transitions may 
play a role. The formalism has also been extended in a fully relativistic treatment [22] and the radial integrals necessary to evaluate the form factors for the tripositive rare-earth ions have been tabulated [23,24] using radial functions from mixed configuration Dirac-Fock atomic calculations. A review of the formalism and neutron scattering experiments involving rare earths can be found in the article by Sinha [25].

III. The Band-Structure Approach.-In the last 10 years there has been dramatic progress in our ability to calculate ground state properties (including magnetization densities). Not only has computing power greatly increased, but also the formalism of Hohenberg, Kohn, and Sham $[26,27]$ has given a practical theoretical foundation for treating interacting electrons in inhomogeneous systems. This must be regarded as a major conceptual advance so that truly first principle calculations are now being performed with no adjustable parameters. At present most of the applications of the formalism make use of band theoretical techniques and the so-called local (spin) density approximation, L(S)DA, for the electronic exchange and correlations energies which enter the theory. Understanding this approximation, its successes and limitations, and how to improve it for the calculation of ground state and excited state phenomena has become a major industry in solid state physics. Recent reviews by Rajagopal on density functional theory [28] and by Koelling on self-consistent band calculations [29] are recommended as is the book on calculated ground state properties of transition metals by Moruzzi, Janak and Williams [30] and the review of the electronic structure of transition metals by Mackintosh and Andersen [31]. There has also been a recent review by Menzinger and Sacchetti [32] on the calculation of spin distributions in transition metal elements and alloys which discusses the LSDA. We will therefore restrict ourselves to a very brief and personal summary of the current situation and possible future directions.

The first principle tight binding calculations of Callaway and Wang for ferromagnetic $\mathrm{Fe}$ and $\mathrm{Ni}[33,34]$ serve to illustrate the success of the band theory approach and have been widely described. Using the form of the LSDA suggested by von Barth and Hedin [35] the calculated magnetic moments (magnetons per atom) were found to be 0.58 for $\mathrm{Ni}$ and 2.16 for $\mathrm{Fe}$ which are to be compared with the experimental spin only values of 0.56 and 2.12 . The corresponding form factors agree to within a few percent with all the measured reflections. There are systematic differences indicating the calculated anisotropy is too smaIl especially for $\mathrm{Ni}$, and that the orbital contribution which was neglected in the calculation is small but significant $[6,36]$. These calculations are we 11 converged and avoid any shape approximations for the potential (such as the muffin-tin approximation). This leaves two major sources of error: the LSDA and the neglect of relativistic effects like spin orbit coupling which is responsible for the small orbital contribution to the magnetization. We briefly describe each of these.

The LSDA of von Barth and Hedin [35] is based on many body calculations for the exchange and correlation energies of a spin polarized electron gas. There has recently appeared accurate Monte Carlo calculations for the electron gas [37] which differ somewhat from earlier approximations. Vosko et al. [38] have reviewed the various approximations and proposed a Pade parameterization of the LSDA based on the Monte Carlo and known high and low density results. This latest local form for the exchange and correlation functionals is worth implementing in future spin polarized calculations since it is probably accurate enough (the error is estimated to be no more than 1 mRy at metallic densities) that one may reasonably assess the importance of non-local effects. There has in fact been some suggestions for approximate non-1ocal exchange-correlation functionals [39-41] but the results have not yet been extended to calculations for ferromagnetic metals.

For materials exhibiting spontaneous magnetization in which the orbital contribution would normally be quenched the presence of spin-orbit coupling results in an orbital magnetic moment and a spectroscopic splitting factor, $g$, 
greater than two. Calculations of the g factor are quite involved and have not been exceptionally successful. For example Singh et al. [42] calculate g=2.14 for $\mathrm{Ni}$ and 2.05 for $\mathrm{Fe}$, while the experimental values are 2.18 and 2.09. The errors are fairly large considering the 2.00 for the spin is "free" and the calculations are for the orbital contributions. Using an earlier and much more approximate band structure Fletcher had already calculated $g=2.14$ for $\mathrm{Ni}$ in 1954 [43]. For $\mathrm{Fe}$ and $\mathrm{Ni}$, orbital form factor calculations are not crucial since the small orbital contribution should be adequately treated with the atomic model if the $g$ factor is known (experimentally if necessary). For heavier elements or those for which the spin density is not close to atomic-like (for example $\mathrm{ZrZn}_{2}$ and $\mathrm{TiBe}_{2}$ [44]) there is no reason to expect the orbital magnetization to be atomic-like. Complete relativistic band structure calculations with a relativistic LSDA [45] and an effective magnetic-scattering amplitude (perhaps similar to what has developed for the atomic model (22]) are still a challenge for theory.

The rare earth metals should also be included under the band theory approach. Although the $4 f$ electrons seem to be well described by the atomic model [36], the ordering of the $4 f$ moments induces a net magnetization in the conduction electrons which is far from atomic-like. Because of the spherical distribution of the half filled $4 \mathrm{f}$ shell, gadolinium is the easiest rare earth metal to study. By subtracting from their. highly precise data an atomic $4 f$ form factor, Moon et al. [46] obtained a conduction electron form factor for gadolinium which did not resemble the form factor for the atomic $5 \mathrm{~d}$ or $6 \mathrm{~s}$ orbitals. The spin-polarized band structure calculations by Harmon and Freeman [47] were successful in qualitatively describing this conduction electron form factor; however the calculations were not self-consistent and quantitative agreement was not obtained. Part of the discrepancy could arise from the subtraction procedure used by Moon et al. where a large atomic form factor (normalized to $\approx 6.42$ ) is subtracted from the large experimental number to yield a conduction electron form factor normalized to $\approx 0.5$. Spin polarized positrion annihilation experiments now in progress [48] may help to clarify the situation since the annihilation matrix elements emphasize the conduction electron states rather than the $4 \mathrm{f}$ orbitals localized near the atomic core. Theoretically it would be desirable to have complete self-consistent band structure calculations which treated both the conduction electron and $4 f$ states simultaneously. Spin polarized, relativistic, self-consistent atomic calculations using the LSDA appeared successful in handling both the $4 f$ and valence electrons [49], however when attempted for the solid the $4 \mathrm{f}$ spin down bands became very slightly occupied [50]. This unphysical result may be associated with the inadequacy of the LSDA to treat the highly inhomogeneous density of the $4 f$ shell. These later calculations did not employ the new relativistic form of the exchange-correlation functionals [45] and avoided spin-orbit coupling to keep spin a good quantum number for the Russell-Saunders coupled $4 \mathrm{f}$ shell. This theoretical picture is sure to advance, motivated by the increased interest in rare earth metals because of mixed valent phenomena and the results of experiments requiring the now available high purity samples.

Finally we only mention the case of molecular crystals containing magnetic transition metal atoms where the magnetization density can reflect the covalent bonding and is valuable for chemical considerations [51].

IV. Induced Magnetization Densities.-The study of the response of metals to an applied magnetic field is nearly as old as quantum mechanics with fundamental understanding of spin [52] and orbital [53-55] susceptibilities as well as a remarkable review by Van Vleck [56] all coming before the discovery of the neutron in 1932. The strategy for arriving at expressions for the susceptibility frequently involves the determination of the partition function and free energy and then taking the second derivative with respect to the magnetic field. This approach has advantages in avoiding difficult problems concerning the behavior of conduction electrons near the surface of crystals [57] and is well suited for incorporating many body effects; however, the resulting expressions can be quite 
intimidating and do not easily lend themselves to physical interpretation or application. The most recent example of this approach is the paper by Misra et a1. [58] and their article contains references to earlier work. Bulk susceptibility measurements provide limited information and agreement with a theoretical result obtained by the evaluation of some complicated formula may involve a fortuitous cancellation of errors. The measurement of the neutron magnetic form factor provides important additional information to test the theory since the various contributions to the induced magnetization density have characteristic spatial distributions.

The Pauli paramagnetic susceptibility arises from the induced unpaired spin up states at the Fermi level. Neglecting spin-orbit coupling the corresponding magnetization is simply a weighted sum of the probability density for states on the Fermi surface [59]

$$
\mathrm{M}_{\text {Pauli }}(\overrightarrow{\mathrm{r}})=2 \mu_{\mathrm{B}}^{2} \int_{\mathrm{E}=\mathrm{E}_{\mathrm{F}}} \frac{\mathrm{dS}(\overrightarrow{\mathrm{k}})}{\left|\nabla_{\mathrm{k}} E(\vec{k})\right|}\left|\psi_{\vec{k}}(\vec{r})\right|^{2}
$$

The spatial distribution for d-like states at the Fermi energy, $\mathrm{E}_{\mathrm{F}}$, is localized when $E_{F}$ is at the top of the $d$ bands (antibonding states) and diffuse when $E_{F}$ falls at the bottom of the $d$ bands (bonding states). Pd and Pt are examples of the former case whose measured form factors $[60,61]$ indicate a magnetization even more localized than the corresponding neutral atomic orbitals, while Sc [62] or Lu [63] exhibit form factors corresponding to very diffuse magnetization densities quite unlike those of the corresponding atoms. Band calculations have been very successful in obtaining normalized form factors in agreement with experiment for these metals [63-66]. To obtain the actual value of the spin contribution the exchange enhancement must be included in the above equation. Recent theoretical advances $[67,68]$ now allow first principle determination of the enhancement accurate to about $10 \%$, so the theoretical situation is indeed in good shape for those metals like Pd for which the spin paramagnetism dominates. The situation for $\mathrm{Sc}$ and $\mathrm{Lu}$ is a bit less clear because the obital contribution is expected to be significant.

In the absence of a magnetic field the orbital angular momentum of Bloch electrons is "quenched" (neglecting spin-orbit coupling), however the applied field induces a first order change in the wavefunctions so that $\left\langle\psi_{k}|\vec{L}| \psi_{k}\right\rangle$ is no longer zero and $a\langle\vec{L}\rangle \cdot \vec{B}$ term in the free energy is produced. This orbital term in the susceptibility [69] can be especially large for transition metals where $\mathrm{E}_{\mathrm{F}}$ falls in the middle of the d-bands $[70]$. It is commonly referred to as the Van Vleck susceptibility. Using the tight binding approximation [59] in which orbital currents are restricted to a single site Carol Theisen (Ph.D. Thesis, Iowa State University, unpublished) evaluated

$$
x^{\perp}=\frac{e^{2} N}{2 m^{2} c^{2}} \quad \sum \frac{f_{n}-f_{n},}{E_{n},-E_{n}}\left|\left\langle n\left|L_{n}\right|_{n^{\prime}}\right\rangle\right|^{2}
$$

for Sc with $\vec{L}$ parallel to the $c$ axis and in the basal plane. She obtained $x_{\mathrm{vV}}=77.8 \mu \mathrm{emu} / \mathrm{mole}$ and $x_{\mathrm{vy}}=95.9 \mu \mathrm{emu} / \mathrm{mole}$. Together with her calculated spin susceptibility enhanced by $4.03[71], \chi_{\text {Pauli }}=299 \mu \mathrm{emu} / \mathrm{mole}$ and a diamagnetic contribution [72] of $-16.6 \mu$ emu/mole she obtained $x^{\|}=360 \mu \mathrm{mu} / \mathrm{mole}$ and $\mathrm{x}^{\perp}$ $=378 \mu \mathrm{emu} / \mathrm{mole}$ which compares favorably with 371 and $384 \mu \mathrm{emu} / \mathrm{mole}$ from experiment [73]. The orbital susceptibility for Lu is expected to be about the same size as for Sc but Lu should have a much smaller spin enhancement so that the orbital contribution should be roughly $50 \%$ of the total. This presents some difficulty since the excellent agreement found between experiment and the spin only theoretical form factor leaves little room for an orbital form factor differing in shape from the pure spin form factor [63]. Stassis et al. suggested the theoretical wavefunctions were not adequate since the calculation was not self-consistent and used a so-called " $\alpha=1$ " form for the exchange potential. A new self-consistent calculation using a better exchange correlation potential [35] gave good agreement with recent de Haas-van Alphen experiments but did not 
yield a substantia11y different form factor [74]. One is left with the suspicion One is left with the suspicion that the orbital form factor for Iu may not be very atomic like. This would be consistent with the observation by oh et al. [59] who calculated the induced orbital form factor for $\mathrm{Cr}$ and found it could be more contracted than an atomic form factor if $\mathrm{E}_{\mathrm{F}}$ fell in the lower part of the d-bands. Unfortunately the work of oh et al, has been the only calculation of an orbital from factor and more evidence needs to be gathered before we can claim to understand orbital from factors as throughly as spin form factors. The need for such calculations is particulariy evident in the case of $\mathrm{Zr}$.

Carol Theisen performed calculations for the magnetic susceptibility of $\mathrm{Zr}$ in the same manner as for those of Sc described above. She obtained $\chi_{\mathrm{vV}}=123.9$ $\mu \mathrm{emu} / \mathrm{mole}, x_{\mathrm{vv}}^{\perp}=131.7 \mathrm{\mu emu} / \mathrm{mole}, x_{\text {diamagnetic }}=-27.2 \mathrm{\mu emu} / \mathrm{mole}$, and $x_{\text {Pauli }}$ enhanced by $1.67[68]=55.8 \mu \mathrm{emu} / \mathrm{mole}$ for a total of $\mathrm{X}^{\|}=153 \mu \mathrm{emu} / \mathrm{mole}$ and $\mathrm{X}^{\perp}=160 \mu \mathrm{emu} / \mathrm{mole}$. These results are about what one might have estimated from Sc with the smaller exchange enhancement for $\mathrm{Zr}$ and larger orbital contribution because $\mathrm{E}_{\mathrm{F}}$ is closer to the center of the $\mathrm{d}$ bands. However the experimental values [75] of $\chi^{\| 1}=149 \mu \mathrm{mu} / \mathrm{mole}$ and $\chi^{\perp}=90 \mu \mathrm{mu} / \mathrm{mole}$ are so different that one must ask if some term is being overlooked. The measured form factor [76] adds to the puzzle since the reflections measured with the field parallel and perpendicular to the $c$ axis do not indicate any anisotropy. A theoretical form factor consisting of a $64 \%$ spin-only band theory form factor and a $36 \%$ atomic orbital form factor fits the neutron data very well and extrapolates to the $x^{1}$ value at zero scattering vector, leaving the large $\chi^{\|}$value unexplained. The evidence seems to implicate anisotropic orbital contributions which contribute to the bulk susceptibility but do not show up for finite scattering vectors. These currents would require calculations going beyond the tight binding approximation described above and by oh et a1. [59].

In trying to use linear response theory to calculate the total orbital susceptiblity in terms of the induced current densities one encounters mathematical difficulties associated with the treatment of operators like $\vec{r} \times \vec{p}$ for infinite crystals or commutations like $[x, H]$ and crystal surfaces. The method employed by Yasui and Shimizu [77] avoids some difficulties by first evaluating the generalized orbital susceptibility, $\chi(q)$, given by Hebborn and March [78] and then numerically taking the limit of $q \rightarrow 0$. Another recent approach which includes orbital contribution beyond the tight binding approximation is based on a real space Green's function method and has been developed by J. Benowitsch and H. Winter (Institut fur Angewandte Kernphysik-I, Kernforschungszentrum, Karlsruhe, to be published). Besides a van vleck orbital contribution they include a term which in the limit of free electrons goes over to the Landau susceptibility. They are able to evaluate this latter term for transition metals and find for $\mathrm{Nb}$ that it is in fact paramagnetic and 5 times larger than what would be expected for free electrons! It would be very interesting to apply either of these methods to hcp elements to see if the dramatic anisotropy differences in Sc and $\mathrm{Zr}$ can be explained. Extending the methods to the evaluation of orbital form factors would not only facilitate comparison with the neutron scattering results but would also help to establish the spatial character of these intercellular currents.

As a summary we may say that the atomic model has probably reached a peak in its formal development, but it will continue to serve as a valuable heuristic aid in the interpretation of form factors. For magnetically ordered elements and compounds self-consistent band theory within the local spin density approximation has proven to be remarkably accurate for calculations of ground state properties such as the equilibrium lattice constant, the bulk modulus, the cohesive energy, the charge density, and the spin part of the magnetization density. There seems to be a small underestimation of anisotropy effects and progress is being made to include relativistic effects in a consistent manner for the treatment of orbital contributions. It is still an open question as to how far band theory or the 
LSDA can be pushed for describing the ground state properties of materials containing rare earths especially in those solids exhibiting mixed valent phenomena. For the case of $\mathrm{CeSn}_{3}$ anyway, the results are encouraging when comparing theory and experiment for de Haas van Alphen orbits $[79,80]$ but 1ess satisfying for the form factor comparison [81-82]. The agreement between band theory and experiment for the form factor of $\mathrm{LaSn}_{3}$ and the lack of agreement for $\mathrm{CeSn}_{3}$ is related to the need for a many body description for the $4 \mathrm{f}$ and conduction electrons [83]. Finally in the area of induced form factors the Pauli paramagnetic spin contribution is being handled routinely while there remains many interesting questions concerning the orbital contribution.

V. Acknowledgments.-The author is grateful to many of his colleagues and collaborators who have contributed to his understanding and enjoyment of research in the area of magnetism. He would like to thank C. Stassis, S. H. Liu, D. D. Koeliing, and A. J. Freeman for many stimulating discussions and support.

This research was sponsored by the Division of Material Sciences, U.S. Department of Energy under Contract No. W-7405-Eng-82. This work was supported by the Director for Energy Research, Office of Basic Energy Sciences.

\section{References}

1. MOON R.M., Intern. J. Magnetism 1 (1971) 219.

2. WATSON R.E. and FREEMAN A.J., Acta. Cryst. 14 (1961) 27.

3. BLUME M., GESCHWIND S. and YAFET Y., Phys. Rev. 181 (1969) 478.

4. SCOTT G.G.; Rev. Mod. Phys. 34 (1962) 102.

5. SHULl C.G. and YAMADA Y., J. Phys. So. Japan 17 Supp1. B-III (1962) 1.

6. VAN LAAR B., MANIAWSKI F., KARPZYK S. and DOBRZYNSKI L., J. Mag. Mag. Mat. 14 (1979) 94.

7. TAPRZYK S., VAN LAAR B. and MANIAWSKI F., J. Mag. Mag. Mat. 23 (1981) 105.

8. BLOCH F., Phys. Rev. 50 (1936) 259.

9. SCHWINGER J., Phys. Rev. 51 (1937) 544.

10. HALPERN 0. and JOHNSON M. H., Phys. Rev. 55 (1939) 898.

11. TRAMMEL G.T., Phys. Rev. 92 (1953) 1387.

12. JOHNSTON D.F., Proc. Phys. Soc. 88 (1966) 37.

13. EDMONDS, A.R., Angular Momentum in Quantum Mechanics (Princeton University Press, Princeton, 1960).

14. JOHNSTON D.F. and RIMMER D.E., J. Phys. C 2 (1969) 1151.

15. I.OVESEY S.B. and RIMMER D.E., Rep. Prog. Phys. 32 (1969) 333.

16. BALCAR E. AND LOVESEY S.W., Phys. Lett. A 31 (1 $\overline{970}$ ) 67 .

17. BALCAR E., LOVESEY S.W. and WEDGEWOOD F.A., J. Phys. C 3 (1970) 1292.

18. MAHENDRA A. and KHAN D.C., J. Phys. C 4 (1971) 3307.

19. MARSHALL $W$. and LOVESEY S.W., Theory of Thermal Neutron Scattering (Oxford University Press, Oxford, 1971).

20. STASSIS C. and DECKMAN H.W., Phys. Rev. B 12 (1975) 1885.

21. STASSIS C. and DECKMAN H.W., J. Phys. C 9 (1976) 2241.

22. STASSIS C. and DECKMAN H.W., Phys. Rev. $\bar{B} 13$ (1976) 4934.

23. STASSIS C., DECKMAN H.W., HARMON B.N., DESCLAUX J.P. and FREEMAN A.J., Phys. Rev. B 15 (1977) 369.

24. FREEMAN A.J. AND DESCLAUX J.P., J. Mag. Mag. Mat. 12 (1979) 11.

25. SINHA S.K., Chapter 7 of Handbook on the Physics and Chemistry of Rare Earths ed. by GSCHNEIDNER K.A. and EYRING L., (North Holland, Amsterdam, $\overline{1978) .}$

26. HOHENBERG P. and KOHN W., Phys. Rev. 136 (1964)B864.

27. KOHN W. and SHAM L.J., Phys. Rev. 140 (1965) Al133.

28. RAJAGOPAL A.K., Adv. in Chem. Phys. 41 (1980).

29. KOELLING D.D., Reports on Progress in Physics 44 (1981) 139.

30. MORUZZI V.L., JANAK J.F., and WILLIAMS A.R., Calculated Electronic Properties of Metals (Pergamon Press, New York, 1978).

31. MACKINTOSH A.R. and ANDERSEN O.K., in Electrons at the Fermi Surface ed. by SPRINGFORD M., (Cambridge University Press, Cambridge, New York, 1980).

32. MENZINGER F. and SACCHETTI F., Nukleonika 24 (1979) 737. 
33. CALlaWAY J. and WANG C.S., Phys. Rev. B 16 (1977) 2095.

34. WANG C.S. and CALLAWAY J., Phys. Rev. B $\overline{15}$ (1977) 298.

35. VON BARTH U, and HEDIN L., J. Phys. C 5 (1972) 1629.

36. MOON R.M., J. de Physique, (to be published, this conference).

37. CEPERLEY D.M. and ALDER B.J., Phys. Rev. Lett. 45 (1980) 566.

38. VOSKO S.H., WILK L. AND NUSAIR M., Can. J. Phys. 58 (1980) 1200.

39. GUNNARSSON O., JONSON M. and LUNDQVIST B.I., Phys. Rev. B 20 (1979) 3136.

40. MACDONALD A.H., LIU K.L., Vosko S.H. and WILK L., Can. J. Phys. 59 (1981) 500 .

41. LANGRETH D.C. and MEHL M.J., Phys. Rev. Lett. 47 (1981) 446.

42. SINGH M., CALLAWAY J. and WANG C.S., Phys. Rev. B 14 (1976) 1214.

43. FLETCHER G.C., Proc. Phys. Soc. A 67 (1954) 505.

44. JARLBORG T., FREEMAN A.J. and KOELAING D.D., J. Mag. Mag. Mat. 23 (1981) 291.

45. RAMANA M.V. and RAJAgOPAL A.K., J. Phys. C 14 (1981) 4291.

46. MOON R.M., KOEHLER W.C., CABLE J.W. and CHILD H.R., Phys. Rev. B 5 (1972) 997.

47. HARMON B.N, and FREEMAN A.J., Phys. Rev. B 10 (1974) 1979.

48. SINCLAIR F., HOFFMAN K.R. and BERKO S., Buli . Amer. Phys. Soc. 26 (1981) 237.

49. KOELLING D.D. and HARMON B.N., J. Phys. C 10 (1977) 3107.

50. HARMON B.N., J. de Physique C5, Supp. 5, $4 \overline{0}$ (1979) 65.

51. FORSYTH J.B., NATO Adv. Study Inst. B 48 (1980) 791.

52. PAULI W., Zeits. f. Physik 41 (1927) 81.

53. LANDAU L., Zeits. f. Physik 64 (1930) 629.

54. TELLER E., Zeits, f. Physik $\overline{67}$ (1930) 311.

55. PEIERLS R., Zeits. f. Physik 80 (1932) 763.

56. VAN VLECK J.H., The Theory of Electric and Magnetic Susceptibilities, (Clarendon Press, Oxford, 1932).

57. PEIERLS R., Surprises in Theoretical Physics, (Princeton University Press, Princeton, 1979) 99.

58. MISRA S.K., MISRA P.K. and MAHANTI S.D., Phys. Rev. B 26 (1982) 1903.

59. OH K.H., HARMON B.N., LIU S.H. and SINHA S.K., Phys. Rev. B 14 (1976) 1283.

60. CABLE J.W., WOLLAN E.O., FLECHER G.P., BRUN T.O. and HORNFEL $\overline{D T}$ S.P., Phys. Rev. Lett. 34 (1975) 281 .

61. MAGLIC R., BRUN T.0., FLECHER G.P. and CHANG Y.K., J. Mag. Mag. Mat. 9 (1978) 318.

62. KOEHLER W.C. and MOON R.M., Phys. Rev. Lett. 36 (1976) 616.

63. STASSIS C., KLINE G.R., LOONG C.-K. and HARMON B.N., Solid State Commun. 23 (1977) 159 .

64. FREEMAN A.J., HARMON B.N. and WATSON-YANG T.J., Phys. Rev. Lett. 34 (1975) 281.

65. WATSON-YANG T.J., FREEMAN A.J. and KOELLING D.D., J. Mag. Mag. Mat. 5 (1977) 277.

66. GUPTA R.P. and FREEMAN A.J., Phys, Rev. Lett. 36 (1976) 613.

67. VOSKO S.H. and PERDEW J.P., Can. J. Phys. 53 (1975) 1385.

68. JANAK J.F., Phys. Rev. B i6 (1977) 255.

69. KUBO R. and OBATA Y., J. Thys. Soc. Japan 11 (1956) 547.

70. PLACE C.M. and RHODES P., Phys. Stat. Sol. (b) 47 (1971) 475.

71. MACDONALD A.H., LIU K.L. and VOSKO S.H., Phys. Rev. B 16 (1977) 777.

72. STASSIS C., Phys. Rev. Lett. 24 (1970) 1415.

73. SPEDDING F.H. and CROAT J.J., J. Chem. Phys. 59 (1973) 2451.

74. TIBBETTS T. and HARMON B.N., Solid State Commun. (in press).

75. COLLINGS E.W. and HO J.C., Phys. Rev. B 4 (1971) 349.

76. STASSIS C., KLINE G.R., HARMON B.N., MOON R.M. and KOEHLER W.C., J. Mag. Mag. Mat. 14 (1979) 303.

77. YASUI M. and SHIMIZU M., J. Phys. F 9 (1979) 1653.

78. HEBBORN J.E. and MARCH N.H., Adv. in Phys. 19 (1970) 175.

79. JOHANSON W.R., CRABTREE G.W., EDELSTEIN A.S, and MCMASTERS D.D., Phys. Rev. Lett. 46 (1981) 504 .

80. KOELLIT̄G D.D., Solid State Commun. 43 (1982) 247.

81. STASSIS C., LOONG C.-K., HARMON B.N., and LIU S.H., J. App1. Phys. 50 (1979) 7567 . 
82. LIU S.H., HARMON B.N., STASSIS C. and SYMEONIDES S., J. Mag. Mag. Mat. 15-18 (1980) 942 .

83. LIU S.H. and HO K.-M., Phys. Rev. B 26 (1982) (in press and to be published). 